\title{
Continuous-time quantum walks on star graphs
}

\author{
S. Salimi * \\ Department of Physics, University of Kurdistan, P.O.Box 66177-15175, Sanandaj, Iran.
}

November 5, 2018

${ }^{*}$ Corresponding author: E-mail addresses: shsalimi@uok.ac.ir 


\begin{abstract}
In this paper, we investigate continuous-time quantum walk on star graphs. It is shown that quantum central limit theorem for a continuous-time quantum walk on star graphs for $N$-fold star power graph, which are invariant under the quantum component of adjacency matrix, converges to continuous-time quantum walk on $K_{2}$ graphs (Complete graph with two vertices) and the probability of observing walk tends to the uniform distribution.

Keywords: Continuous-time quantum walk, Star graph, Spectral distribution.
\end{abstract}

PACs Index: 03.65.Ud 


\section{Introduction}

Quantum walks were introduced in the early 1990s by Aharonovich, davidovich and Zaggury [1]. Since then the topic has attracted considerable interest. The continuing attraction can be traced back to at least two reasons. First, the quantum walk is of sufficient interest in its own right because there are fundamental differences compared to the classical random walk. Next, quantum walks offer quite a number of possible applications. One of the best known is the link between quantum walks and quantum search algorithms which are superior to their classical counterparts[2,3]. Similar to classical random walk there are two types of quantum walks, discrete and continuous time [1,4]. A study of quantum walks on simple graph is well known in physics (for more details see [5]). Recent studies of quantum walks on more general graphs were described in $[2,6,7,8,9,10,11,12]$. Some of these works studies the problem in the important context of algorithmic problems on graphs and suggests that quantum walks is a promising algorithmic technique for designing future quantum algorithms. One approach for investigation of continuous-time quantum walk (CTQW) on graphs is using the spectral distribution associated with the adjacency matrix of graphs $[13,14,15,16,17,18,19]$. Authors in Refs. $[13,14]$ have introduced a new method for calculating the probability amplitudes of quantum walk based on spectral distribution. In this method a canonical relation between the Fock space of stratification graph and set of orthogonal polynomials has been established where leads to obtain the probability measure (spectral distribution) adjacency matrix graph. The method of spectral distribution only requires simple structural data of graph and allows us to avoid a heavy combinational argument often necessary to obtain full description of spectrum of the adjacency matrix.

In this paper we investigate CTQW on star graphs, which are discussed by Burioni et, al. $[20,21]$ as models of the Bose-Einstein condensation, by quantum probability theory. To this aim, first by turning the graph into metric space based on distance function, we have been able 
to stratify and quantum decomposition $[13,23]$, such that the basis of Hilbert space of quantum walk consist of superposition of quantum kets of vertices belonging to the same stratum with the same coefficient (Fock space). Then by applying an isomorphism from Fock space onto the closed linear span of the orthogonal polynomials with respect to the measure $\mu$ we obtain spectral distribution adjacency matrix graph and probability amplitudes of observing walk at strata. Also we try to investigate quantum central limit theorem for CTQW in $N$-fold star power graphs as $N \longrightarrow \infty$, and show the CTQW converges to CTQW on $K_{2}$ (Complete graph with two vertices) where the probability of observing walk tends to the uniform distribution. On the other hand, quantum walker induces to the graph that spectral distribution it tend to the Bernoulli distribution $\mu \longrightarrow \frac{1}{2}\left(\delta_{1}+\delta_{-1}\right)$.

The organization of the paper is as follows: we give a brief review of graph, star graph, stratification and quantum decomposition in the section 2. Section 3 is devoted to study CTQW for some example of star graphs via quantum probability theorey and try to investigate quantum central limit theorem for CTQW on their graphs. In the conclusion we summarize the obtained results and discuss possible development. Finally, in the appendix the determination of spectral distribution associated with adjacency matrix by Stieltjes transform is derived.

\section{Star graph, Stratification and Quantum decomposi- tion}

Let $V$ be a non-empty set and $E$ be a subset of $\{\{\alpha, \beta\} \mid \alpha, \beta \in V$ for $\alpha \neq \beta\}$. The pair $G=(V, E)$ is called a graph, where elements of $V$ and $E$ are vertices and edges of graph, respectively. We say that two vertices of $\alpha$ and $\beta$ are adjacent if $\{\alpha, \beta\} \in E$ and write $\alpha \sim \beta$. A finite sequence $\alpha_{0}, \alpha_{1}, \ldots, \alpha_{n}$ is said a walk of length $n$ if $\alpha_{k} \sim \alpha_{k+1}$ for $k=0,1, \ldots, n-1$. A graph is called connected if any pair of distinct vertices is connected by a walk. The degree or 
valency of a vertex $\alpha \in V$ is defined by

$$
\kappa(\alpha)=|\{\beta \in V \mid \beta \sim \alpha\}|
$$

where |.| denote the cardinality. For a graph $G$ the adjacency matrix $A$ is given by

$$
A_{\alpha \beta}= \begin{cases}1 & \text { if } \alpha \sim \beta \\ 0 & \text { otherwise. }\end{cases}
$$

Obviously, (i) $A$ is a symmetric (ii) elements of $A$ take a value in $\{0,1\}$ (iii) diagonal elements of $A$ are 0 . Conversely, for a non-empty set $V$, a structure graph is uniquely determined by a such matrix which indexed by $V$. On the other hand, $A$ is considered as an operator acting on the Hilbert space $l^{2}(V)$ in such a way that

$$
A|\alpha\rangle=\sum_{\alpha \sim \beta}|\beta\rangle, \quad \alpha \in V,
$$

where $\{|\alpha\rangle \mid \alpha \in V\}$ forms a complete orthogonal basis of $l^{2}(V)$.

In this paper, we focus on investigating CTQW on star graphs which are also discussed by Burioni et al. [20, 21] as models of the Bose- Einstein condensation. A star graph is obtained from star product of graphs which we define in the following.

Let us consider two graphs $G^{\nu}, \nu=1,2$ with adjacency matrices $A^{\nu}$, each of which is equipped with an origin $o_{\nu} \in V^{(\nu)}$. Define a matrix $A$ as

$$
A_{(\alpha, \beta),(\dot{\alpha}, \dot{\beta})}=A_{\alpha \dot{\alpha}}^{(1)} \delta_{\beta o_{2}} \delta_{\dot{\beta} o_{2}}+\delta_{\alpha o_{1}} \delta_{\dot{\alpha} o_{1}} A_{\beta \dot{\beta}}^{(2)}, \quad \alpha, \dot{\alpha} \in V^{(1)}, \quad \beta, \dot{\beta} \in V^{(2)}
$$

We observe that $A$ is symmetric, elements take a value in $\{0,1\}$ and the diagonal consists of 0 . Therefore $A$ is the adjacency matrix of a graph. The connected component containing $\left(o_{1}, o_{2}\right)$ is called the star product of $G^{(1)}$ and $G^{(2)}$ and the resulted graph is a star graph [23](see Fig.1). One can generalize this definition for $\nu=1,2, \ldots, N$ that we consider $N$-fold star power graphs for our aims. The stratification is introduced $[13,23]$ by taking $o=\left(o_{1}, o_{2}, \ldots, o_{N}\right)$ as the origin and have

$$
V=\bigcup_{k=0}^{\infty} V_{k}, \quad V_{i}=\{\alpha \in V \mid \partial(o, \alpha)=k\}
$$


Here $\partial(\alpha, \beta)$ stands for the length of the shortest walk connecting $\alpha$ and $\beta$. According to the stratification (2-3), we define a unit vector by

$$
\left|\phi_{k}\right\rangle=\frac{1}{\sqrt{\left|V_{k}\right|}} \sum_{\alpha \in V_{k}}|k, \alpha\rangle
$$

where $|k, \alpha\rangle$ denotes the eigenket of the $\alpha$-th vertex at the stratum $k$ and let $\Gamma(G)$ the closed subspace of $l^{2}(V)$ spanned by $\left\{\left|\phi_{k}\right\rangle\right\}$. Moreover, the stratification (2-3)give rise to a quantum decomposition:

$$
A=A^{+}+A^{-}+A^{0}
$$

If $\Gamma(G)$ is invariant under the actions of the quantum components quantum components $A^{\varepsilon}$, $\varepsilon \in\{+,-, 0\}$, then there exist two Szegö- Jacobi sequences $\left\{\omega_{k}\right\}_{k=1}^{\infty}$ and $\left\{\alpha_{k}\right\}_{k=1}^{\infty}$ derived from $A$, such that

$$
\begin{gathered}
A^{+}\left|\phi_{k}\right\rangle=\sqrt{\omega_{k+1}}\left|\phi_{k+1}\right\rangle, \quad k \geq 0 \\
A^{-}\left|\phi_{0}\right\rangle=0, \quad A^{-}\left|\phi_{k}\right\rangle=\sqrt{\omega_{k}}\left|\phi_{k-1}\right\rangle, \quad k \geq 1 \\
A^{0}\left|\phi_{k}\right\rangle=\alpha_{k+1}\left|\phi_{k}\right\rangle, \quad k \geq 0 .
\end{gathered}
$$

The above coefficients (i.e., Szegö- Jacobi sequences ) are obtained from geometric feature of graph $[13,23]$. Then $\left(\Gamma(G), A^{+}, A^{-}, A^{o}\right)$ is an interacting Fock space associated with SzegöJacobi sequences $\left\{\omega_{k}, \alpha_{k}\right\}$.

\section{CTQW on star graph via quantum probability theory}

In the study of CTQW on graphs, the spectral distribution of $A$ in the vacuum state $\left|\phi_{0}\right\rangle$ plays an essential role [13], which is by definition a probability distribution $\mu$ uniquely specified by

$$
\left\langle A^{m}\right\rangle=\left\langle\phi_{0}\left|A^{m}\right| \phi_{0}\right\rangle=\int x^{m} \mu(d x), \quad m=0,1,2, \ldots,
$$

where, according to $[13,14,23],\left\langle A^{m}\right\rangle$ coincides with the number of $m$-step walks starting and terminating at origin point $o$. For analyzing the spectral distribution $\mu$ of adjacency matrix 
$A$, we use the method of quantum decomposition which is a powerful tool. The spectral distribution $\mu$ is determined by applying the canonical isomorphism from the interacting Fock space onto the closed linear span of orthogonal polynomials determined by Szegö- Jacobi sequences $\left\{\omega_{k}, \alpha_{k}\right\}$. In fact the determination of $\mu$ is the main problem in the spectral theory of operators, where in the case of star graphs is quite possible by using the Stieltjes method, as it is explained in appendix A. Then by using the quantum decomposition relations (2-5)-(2-8) and the recursion relation of polynomials $P_{n}(n)(\mathrm{A}-1)$, the other matrix elements as

$$
\left\langle\phi_{k}\left|A^{m}\right| \phi_{0}\right\rangle=\frac{1}{\sqrt{\omega_{1} \omega_{2} \ldots \omega_{k}}} \int x^{m} P_{k}(x) \mu(d x), \quad m=0,1,2, \ldots
$$

One of our main goals in this paper is the evaluation of probability amplitudes of CTQW by using Eq.(3-10), such that we have

$$
q_{k}(t)=\left\langle\phi_{k}\left|e^{-i t A}\right| \phi_{0}\right\rangle=\frac{1}{\sqrt{\omega_{1} \omega_{2} \ldots \omega_{k}}} \int e^{-i t x} P_{k}(x) \mu(d x)
$$

where $\left|q_{k}(t)\right|^{2}$ is the probability of observing the walk at the stratum $k$ at time $t$. The conservation of probability $\sum_{k}\left|q_{k}(t)\right|^{2}=1$ follows immediately from Eq. (3-11) by using the completeness relation of orthogonal polynomials $P_{n}(x)$. In the appendix $A$ reference [13] is provided the walker has the same probability at the all sites belonging to the same stratum, i.e., we have $\left|q_{i k}(t)\right|^{2}=\frac{\left|q_{k}(t)\right|^{2}}{\left|V_{k}\right|}$, for $i \in V_{k}$, where $\left|q_{i k}(t)\right|^{2}$ denotes the probability of the walker at the $i$-th vertex of $k$-th stratum $V_{k}$. Investigation of CTQW via spectral distribution method, which is introduced as a new method for calculating the probability amplitudes quantum walk (for more details see [13] ), allows us to avoid a heavy combinational argument often necessary to obtain full description of spectrum of the Hamiltonian.

We can now investigate CTQW on star graphs. In the first, we calculate CTQW on two simple star graph and study quantum central limit theorem for them. In the end, we study this question for star lattice. 


\subsection{Examples 1.}

In this example we investigate the CTQW on the star finite path graph with three vertices as Fig.2. Then we have

$$
\omega_{1}=N, \quad \omega_{2}=1 ; \quad \alpha_{n}=0
$$

and

$$
G_{\mu_{N}}(z)=\frac{z^{2}-1}{z^{3}-(N+1) z}, \quad \mu_{N}=\frac{1}{N+1} \delta_{0}+\frac{N}{2(N+1)}\left(\delta_{\sqrt{N+1}}+\delta_{-\sqrt{N+1}}\right) .
$$

Therefore the amplitudes probability of CTQW on this graph are as follows:

$$
\begin{gathered}
q_{0}(t)=\int e^{-i x t} \mu(d x)=\frac{1}{N+1}[1+N \cos (\sqrt{N+1} t)] \\
q_{1}(t)=\frac{1}{\sqrt{N}} \int x e^{-i x t} \mu(d x)=-i \sqrt{\frac{N}{N+1}} \sin (\sqrt{N+1} t) \\
q_{2}(t)=\frac{1}{\sqrt{N}} \int\left(x^{2}-N\right) e^{-i x t} \mu(d x)=\frac{N}{(N+1) \sqrt{N}}[-1+\cos (\sqrt{N+1} t)] .
\end{gathered}
$$

\section{Quantum central limit theorem}

Having studied CTQW on this graph for $N$ arbitrary, we investigate CTQW in the limit of large $N \longrightarrow \infty$ which in fact we discuss this question as a quantum central limit theorem for CTQW. To state a quantum central limit theorem for CTQW we have[16]

$$
q_{k}(t)=\lim _{N \longrightarrow \infty}\left\langle\phi_{k}\left|e^{\frac{-i t A}{\sqrt{N}}}\right| \phi_{0}\right\rangle=\lim _{N \longrightarrow \infty} \frac{1}{\sqrt{\omega_{1} \omega_{2} \ldots \omega_{k}}} \int P_{k}(x) e^{-i x t / \sqrt{N}} \mu_{N}(x) d x
$$

Then we obtain the amplitudes probability as

$$
\begin{gathered}
q_{0}(t)=\lim _{N \longrightarrow \infty} \int_{R} e^{\frac{-i t x}{\sqrt{N}}} \mu(d x)=\lim _{N \longrightarrow \infty} \int_{R} e^{\frac{-i t x}{\sqrt{N}}}\left(\frac{1}{N+1} \delta_{0}+\frac{N}{2(N+1)}\left(\delta_{\sqrt{N+1}}+\delta_{-\sqrt{N+1}}\right)\right) d x \\
=\frac{1}{2}\left(e^{-i t}+e^{i t}\right)=\cos t \\
q_{1}(t)=\lim _{N \longrightarrow \infty} \frac{1}{\sqrt{N}} \int_{R} x e^{\frac{-i t x}{\sqrt{N}}} \mu(d x)= \\
\lim _{N \longrightarrow \infty} \frac{1}{\sqrt{N}} \int_{R} x e^{\frac{-i t x}{\sqrt{N}}}\left(\frac{1}{N+1} \delta_{0}+\frac{N}{2(N+1)}\left(\delta_{\sqrt{N+1}+\delta_{-\sqrt{N+1}}}\right) d x\right.
\end{gathered}
$$




$$
\begin{aligned}
\frac{1}{2}\left(e^{-i t}-e^{i t}\right) & =-i \sin t, \\
q_{2}(t) & =0,
\end{aligned}
$$

where the probability of the observing walk $\left(\left|q_{i}(t)\right|^{2}\right.$, for $\left.i=0,1\right)$ reaches the uniform distribution periodically at $t=k \pi+\frac{\pi}{4}$ for $k \in Z$.

Indeed, in the limit of large $N \longrightarrow \infty$ (e.i., quantum central limit theorem ), the CTQW on this graph is reduced to CTQW on $K_{2}$ (Complete graph with two vertices). In other words, the spectral distribution $\mu_{N}$ associated with the adjacency matrix of the graph tend to the Bernoulli distribution $\left(\mu_{N} \longrightarrow \frac{1}{2}\left(\delta_{1}+\delta_{-1}\right)\right)$, where is the spectral distribution of $K_{2}$ graphs.

\subsection{Examples 2.}

In the second example we consider CTQW on the star graph as Fig.3. Then we have

$$
\omega_{1}=2 N, \quad \omega_{2}=2 ; \quad \alpha_{n}=0
$$

and

$$
G_{\mu_{N}}(z)=\frac{x^{2}-2}{x\left(x^{2}-2(N+1)\right)}, \quad \mu_{N}=\frac{1}{N+1} \delta_{0}+\frac{N}{2(N+1)}\left(\delta_{\sqrt{2(N+1)}}+\delta_{-\sqrt{2(N+1)}}\right) .
$$

The amplitudes probability of CTQW on this graph are as:

$$
\begin{gathered}
q_{0}(t)=\int e^{-i x t} \mu(d x)=\frac{1}{N+1}[1+N \cos (\sqrt{2(N+1)} t)] \\
q_{1}(t)=\frac{1}{\sqrt{2 N}} \int x e^{-i x t} \mu(d x)=-i \sqrt{\frac{N}{N+1}} \sin (\sqrt{2(N+1)} t) \\
q_{2}(t)=\frac{1}{2 \sqrt{N}} \int\left(x^{2}-N\right) e^{-i x t} \mu(d x)=\frac{N}{2(N+1) \sqrt{N}}[-1+2 \cos (\sqrt{N+1} t)] .
\end{gathered}
$$

Also in this example, quantum central limit theorem for CTQW is reduced on CTQW on complete graph $K_{2}$. 


\subsection{Examples 3.}

\section{Star Lattice}

The star lattice is the $N$-fold star power of finite path graph, see Fig.4. One can show that the two sequence $\left\{\omega_{k}\right\}$ and $\left\{\alpha_{k}\right\}$ obtain as

$$
\omega_{1}=N, \quad \omega_{2}=\omega_{3}=\cdots=1 ; \quad \alpha_{k}=0
$$

Substituting sequence $\omega_{k}$ and $\alpha_{k}$ in (5.22), the Stieltjes transform $G_{\mu_{N}}(z)$ of spectral distribution $\mu_{N}$ takes the following form

$$
G_{\mu_{N}}(z)=\frac{1}{z-\frac{N}{z-\frac{1}{z-\frac{1}{z-\frac{1}{\ddots}}}}}
$$

In order to evaluate the continued fraction, we need first to evaluate the following infinite continued fraction defined as

$$
\tilde{G}_{\mu}(z)=\frac{1}{z-\frac{1}{z-\frac{1}{z-\frac{1}{z-\frac{1}{\ddots}}}}}=\frac{1}{z-\tilde{G}_{\mu}(z)},
$$

where by solving above equation we have

$$
\tilde{G}_{\mu}(z)=\frac{1}{2}\left(z-\sqrt{z^{2}-4}\right)
$$

Then by substituting (3-25) in (3-31), we obtain the following expression for the Stieltjes transform of $\mu$

$$
G_{\mu_{N}}(z)=\frac{1}{z-N \tilde{G}_{\mu}(z)}=\frac{1}{2} \frac{(2-N) z-N \sqrt{z^{2}-4}}{N^{2}-(N-1) z^{2}} .
$$

Finally by applying Stieltjes inversion formula, we acquire the absolutely continuous part of spectral distribution $\mu_{N}$ as follows

$$
\mu_{N}(x)=\frac{1}{2 \pi} \frac{N \sqrt{4-x^{2}}}{N^{2}-(N-1) x^{2}} ; \quad-2 \leq x \leq 2 .
$$


Now we investigate CTQW on some of the known infinite graphs can be obtained from star lattice by appropriate choice of $N$ as:

A. For $N=1$ we obtain finite path graph. Then we have

$$
\begin{aligned}
& \omega_{1}=1, \quad \omega_{2}=\omega_{3}=\cdots=1 ; \quad \alpha_{k}=0 \\
& \mu_{1}(x)=\frac{1}{2 \pi} \sqrt{4-x^{2}} ; \quad-2 \leq x \leq 2,
\end{aligned}
$$

where the amplitudes probability for CTQW on this graph are

$$
q_{k}(t)=i^{k}\left(J_{k}(t)+J_{k+2}(t)\right)=2 i^{k}(k+1) \frac{J_{k+1}(t)}{t} ; \quad k=0,1, \ldots
$$

(for more detail see Ref.[13]).

B. For $N=2$ we obtain infinite line graph where the two sequences, spectral distribution and the amplitude probability for observing walk at strata at time $t$ are

$$
\begin{gathered}
\omega_{1}=2, \quad \omega_{2}=\omega_{3}=\cdots=1 ; \quad \alpha_{k}=0 \\
\mu_{2}(x)=\frac{1}{\pi} \frac{1}{\sqrt{4-x^{2}}} ; \quad-2 \leq x \leq 2, \\
q_{0}(t)=J_{0}(t) ; \quad q_{k}(t)=i^{k} \sqrt{2} J_{k}(t), \quad k \geq 1,
\end{gathered}
$$

(for more detail see Ref.[13]).

C. For $N \geq 3$ the total mass of $\mu_{N}(x)$ is less than one (i.e., $\int \mu_{N}(x) d x \leq 1$ ). In fact, it contains a discrete measure where by a simple computation one can obtain as [24]

$$
\mu_{N}(x)=\frac{1}{2 \pi} \frac{N \sqrt{4-x^{2}}}{N^{2}-(N-1) x^{2}}+\frac{N-2}{2 N-2}\left(\delta_{\frac{N}{\sqrt{N-1}}}+\delta_{\frac{-N}{\sqrt{N-1}}}\right) ; \quad-2 \leq x \leq 2 .
$$

\section{Quantum central limit theorem}

Now we consider behavior CTQW on this graph for large $N$ (i.e., $N \longrightarrow \infty$ ) where we discuss this question as a quantum central limit theorem for CTQW.

The quantum central limit theorem for CTQW is

$$
q_{k}(t)=\lim _{N \longrightarrow \infty}\left\langle\phi_{k}\left|e^{-i A t / \sqrt{N}}\right| \phi_{0}\right\rangle=\lim _{N \longrightarrow \infty} \frac{1}{\sqrt{N}} \int P_{k}(x) e^{-i x t / \sqrt{N}} \mu_{N}(x) d x
$$




$$
\begin{gathered}
=\lim _{N \rightarrow \infty} \frac{1}{\sqrt{N}} \int P_{k}(x) e^{-i x t / \sqrt{N}}\left(\frac{1}{2 \pi} \frac{N \sqrt{4-x^{2}}}{N^{2}-(N-1) x^{2}}+\frac{N-2}{2 N-2}\left(\delta_{\frac{N}{\sqrt{N-1}}}+\delta_{\frac{-N}{\sqrt{N-1}}}\right)\right) d x \\
=\lim _{N \rightarrow \infty} \frac{1}{\sqrt{N}} \int P_{k}(x) e^{-i x t / \sqrt{N}} \frac{N-2}{2 N-2}\left(\delta_{\frac{N}{\sqrt{N-1}}}+\delta_{\frac{-N}{\sqrt{N-1}}}\right) d x \\
=\lim _{N \rightarrow \infty} \frac{N-2}{2 N-2}\left(\frac{P_{k}\left(\frac{N}{\sqrt{N-1}}\right)}{\sqrt{N}} e^{\left.\frac{-i t N}{\sqrt{N(N-1)}}+\frac{P_{k}\left(\frac{-N}{\sqrt{N-1}}\right)}{\sqrt{N}} e^{\frac{i t N}{\sqrt{N(N-1)}}}\right)}\right.
\end{gathered}
$$

By a simple computation one can show that $\lim _{N \rightarrow \infty} \frac{P_{k}\left(\frac{ \pm N}{\sqrt{N-1}}\right)}{\sqrt{N}}=0$ for $k \geq 2$. Then we have

$$
\begin{gathered}
q_{0}(t)=\frac{1}{2}\left(e^{-i t}+e^{i t}\right)=\cos t \\
q_{1}(t)=\frac{1}{2}\left(e^{-i t}-e^{i t}\right)=-i \sin t \\
q_{k}(t)=0 \quad \text { for } \quad k \geq 2
\end{gathered}
$$

We see that quantum central limit theorem for CTQW on the star lattice is reduced on complete graph $K_{2}$ and the amplitudes probability reach the uniform distribution. Really, for every star graph that is invariant under the quantum component $A^{\epsilon}, \epsilon \in\{+,-, o\}$, one can show that quantum central limit theorem for CTQW on it is reduced CTQW on complete graph $K_{2}$.

\section{Conclusion}

In this work, we have investigated CTQW on star graphs, which are discussed by Burioni et, al. [20, 21] as models of the Bose-Einstein condensation, by quantum probability theory. First, we have equipped graph to metric based on distance function. Then we have stratified graph and according to stratification accomplished quantum decomposition of adjacency matrix. The basis of Hilbert space of quantum walk form a Fock space where by applying an isomorphism from it onto the closed linear span of the orthogonal polynomials we have obtained spectral distribution adjacency matrix graph and probability amplitudes of observing walk at strata. Also, we have studied quantum central limit theorem for CTQW on star graphs and shown the CTQW converges to CTQW on $K_{2}$ (complete graph with two vertices), such that the probability of observing walk tend to the uniform distribution. Indeed, we can generalize CTQW 
on star graphs which are invariant under the quantum component $A^{\epsilon}, \epsilon \in\{+,-, o\}$, quantum central limit theorem for them induce in CTQW on graphs that the spectral distribution it tend to the Bernoulli distribution.

\section{Appendix A}

\section{Determination of spectral distribution by the Stieltjes transform}

In this appendix we explain how we can determine spectral distribution $\mu(x)$ of the graphs, by using the Szegö-Jacobi sequences $\left(\left\{\omega_{k}\right\},\left\{\alpha_{k}\right\}\right)$. To this aim we may apply the canonical isomorphism from the interacting Fock space onto the closed linear span of the orthogonal polynomials determined by the Szegö-Jacobi sequences $\left(\left\{\omega_{i}\right\},\left\{\alpha_{i}\right\}\right)$. More precisely, the spectral distribution $\mu$ under question is characterized by the property of orthogonalizing the polynomials $\left\{P_{n}\right\}$ defined recurrently by

$$
\begin{gathered}
P_{0}(x)=1, \quad P_{1}(x)=x-\alpha_{1}, \\
x P_{n}(x)=P_{n+1}(x)+\alpha_{n+1} P_{n}(x)+\omega_{n} P_{n-1}(x),
\end{gathered}
$$

for $n \geq 1$.

As it is shown in [25], the spectral distribution can be determined by the following identity:

$$
G_{\mu}(z)=\int_{R} \frac{\mu(d x)}{z-x}=\frac{1}{z-\alpha_{1}-\frac{\omega_{1}}{z-\alpha_{2}-\frac{\omega_{2}}{z-\alpha_{3}-\frac{\omega_{3}}{z-\alpha_{4}-\cdots}}}}=\frac{Q_{n-1}^{(1)}(z)}{P_{n}(z)}=\sum_{l=1}^{n} \frac{A_{l}}{z-x_{l}},
$$

where $G_{\mu}(z)$ is called the Stieltjes transform and $A_{l}$ is the coefficient in the Gauss quadrature formula corresponding to the roots $x_{l}$ of polynomial $P_{n}(x)$ and where the polynomials $\left\{Q_{n}^{(1)}\right\}$ are defined recurrently as

$Q_{0}^{(1)}(x)=1$,

$Q_{1}^{(1)}(x)=x-\alpha_{2}$,

$x Q_{n}^{(1)}(x)=Q_{n+1}^{(1)}(x)+\alpha_{n+2} Q_{n}^{(1)}(x)+\omega_{n+1} Q_{n-1}^{(1)}(x)$,

for $n \geq 1$. 
Now if $G_{\mu}(z)$ is known, then the spectral distribution $\mu$ can be recovered from $G_{\mu}(z)$ by means of the Stieltjes inversion formula:

$$
\mu(y)-\mu(x)=-\frac{1}{\pi} \lim _{v \rightarrow 0^{+}} \int_{x}^{y} \operatorname{Im}\left\{G_{\mu}(u+i v)\right\} d u .
$$

Substituting the right hand side of (A-2) in (A-3), the spectral distribution can be determined in terms of $x_{l}, l=1,2, \ldots$, the roots of the polynomial $P_{n}(x)$, and Guass quadrature constant $A_{l}, l=1,2, \ldots$ as

$$
\mu=\sum_{l} A_{l} \delta\left(x-x_{l}\right)
$$

( for more details see Refs. [13, 14, 25, 26].)

\section{References}

[1] Y. Aharonov, L. Davidovich, N. Zagury, Phy. Rev. lett 48 (1993)1687.

[2] A. Childs, E. Deotto, R. Cleve, E. Farhi, S. Gutmann, D. Spielman, Proc. 35th Ann. Symp. Theory of Computing (ACM Press) 59 (2003).

[3] N. Shenvi, J. Kempe, K. B. Whaley,Phys. Rev. A 67 (2003) 052307.

[4] J. Kempe, Contemporary Physics 44 (2003) 307.

[5] R. Feynman, R. Leighton, M. Sands, The Feynman Lectures on Physics, Volume 3, Addison-Wesley, 1965.

[6] E. Farhi, S. Gutmann, Phys. Rev. A 58 (1998) 915.

[7] E. Farhi, M. Childs, S. Gutmann, Quantum Information Processing 1 (2002) 35.

[8] A. Ambainis, E. Bach, A. Nayak, A. Viswanath, J. Watrous , Proceedings of the 33rd ACM Annual Symposium on Theory Computing (ACM Press), 2001. 
[9] D. Aharonov, A. Ambainis, J. Kempe, U. Vazirani, Proceedings of the 33rd ACM Annual Symposium on Theory Computing (ACM Press), 2001.

[10] C. Moore, A. Russell, Proceedings of the 6th Int. Workshop on Randomization and Approximation in Computer Science (RANDOM'02), 2002.

[11] J. Kempe, Proceedings of 7th International Workshop on Randomization and Approximation Techniques in Computer Science (RANDOM'03), 2003.

[12] H. Krovi, Todd A. Brun, Phys. Rev. A 75 (2007) 062332.

[13] M. A. Jafarizadeh, S. Salimi, Ann. Phys 322 (2007) 1005.

[14] M. A. Jafarizadeh, S. Salimi, J. Phys. A 39 (2006) 1.

[15] M. A. Jafarizadeh, R. Sufiani, S. Salimi, S. Jafarizadeh, Eur. Phys. J. B 59 (2007) 199.

[16] N. Konno, Inf. Dim. Anal. Quantum Probab. Rel. Topics 9 (2006) 287.

[17] N. Konno, International Journal of Quantum Information 4 (2006)1023.

[18] S. Salimi, e-print quan-ph/07105813.

[19] S.Salimi, e-print quan-ph/07103043.

[20] R. Burioni, D. Cassi, I. Meccoli, M. Rasetti, S. Regina, P. Sodano and A. Vezzani, Europhys. Lett., 52 (2000) 251256 .

[21] R. Burioni, D. Cassi, M. Rasetti, P. Sodano and A. Vezzani, J. Phys. B: At. Mol. Opt. Phys., 34 (2001) 46974710.

[22] J. L. Gross, T. W. Tucker, Topological Graph Theory, Wiley Interscience, New York, 1987.

[23] N. Obata, Interdisciplinary Information Sciences 10 (2004) 41. 
[24] N. Obata, Notions of independence in quantum probability and spectral analysis of graphs, to appear in Sugaku Expositions.

[25] T. S. Chihara, An Introduction to Orthogonal Polynomials, London: Gordon and Breach, 1978.

[26] J. A. Shohat, J. D. Tamarkin, The Problem of Moments, American Mathematical Society, Providence, RI, 1943. 
Figure Captions

Figure-1: Star graph.

Figure-2: Figure.2

Figure-3: Figure.3.

Figure-4: Star lattice for $N=8$ 